\title{
Improving cardiac SPECT accuracy: Old robustness for a new gold standard
}

\author{
Alessia Gimelli, MD, a and Riccardo Liga, $M D^{b}$ \\ a Fondazione Toscana Gabriele Monasterio, Pisa, Italy \\ ${ }^{\text {b }}$ University Hospital of Pisa, Pisa, Italy
}

Received Jan 27, 2016; accepted Jan 27, 2016

doi: 10.1007/s12350-016-0435-1

\section{See related article, pp. 672-682}

Medical diagnostics is based on the ability to correctly discriminate a pathologic finding from normality. In order to accomplish this task, a clear definition of normality, possibly through an accepted diagnostic "gold standard," is required. However, despite the simplicity of this general concept, in many pathologies the definition of a normal state may be exceedingly difficult, making the entire diagnostic process somehow obscure.

Ischemic heart disease (IHD) represents a classical example of how the definition of normality may vary considerably according to the global understanding of the pathology. As a matter of fact, for decades, the occurrence of IHD has been correlated with the presence of anatomically obstructive epicardial coronary stenosis (i.e., able to reduce coronary diameter of more than 50 or $70 \%) .{ }^{1,2}$ Accordingly, normality was classically identified by the absence of anatomically obstructive coronary artery disease (CAD) and the accuracy of each non-invasive imaging modality, such as single-photon emission computed tomography (SPECT), has been tested against an anatomic gold standard (i.e., invasive coronary angiography, ICA). ${ }^{3-5}$

However, solid evidence has demonstrated that the presence of anatomically significant CAD is a poor predictor of the development of myocardial ischemia, since relevant alteration of myocardial perfusion might develop also in patients with non-critical coronary

\footnotetext{
Reprint requests: Alessia Gimelli, Fondazione Toscana Gabriele Monasterio, Via Moruzzi 1, 56124, Pisa, Italy; gimelli@ftgm.it J Nucl Cardiol 2017;24:683-6.

$1071-3581 / \$ 34.00$

Copyright (C) 2016 American Society of Nuclear Cardiology.
}

stenosis, or even in the absence of appreciable coronary abnormalities. ${ }^{6-8}$

In order to overcome these limitations, in the last decade, the reference for the diagnosis of IHD has radically changed from an anatomical one (i.e., a coronary stenosis evident on angiography) to an anatomo-functional one (i.e., an ischemia-causing coronary lesion). ${ }^{9-11}$ The gold standard itself for the diagnosis of IHD has also radically changed from a simply anatomical test, as represented by ICA, to an anatomofunctional one, such as ICA plus fractional flow reserve (FFR). ${ }^{9,12}$ In line with these evidences, it is now widely accepted that an ischemia-causing coronary stenosis should be treated aggressively, based on superior clinical and prognostic data, while, in the absence of ischemia, a conservative management of CAD may be safely performed. ${ }^{9}$

Despite this relevant change, the accuracies of all the currently used non-invasive imaging modalities in detecting the presence of IHD have been obtained against ICA, ultimately depicting their diagnostic power in unmasking the presence of anatomically significant CAD.

In this respect, myocardial perfusion imaging (MPI) with SPECT still represents the backbone for the noninvasive evaluation of regional myocardial perfusion, giving the change to obtain accurate measures of myocardial ischemia. ${ }^{3,13}$ Through the use of extensively validated quantification perfusion software, a rapid and simple semi-quantification of myocardial ischemic burden is feasible, further increasing the overall test's accuracy and reproducibility. ${ }^{14}$ As obvious, these softwares depend on the existence of a pre-installed normal limits database of myocardial perfusion, generally created using the SPECT data of a relatively limited population of patients at exceedingly low risk of CAD (i.e., $<5 \%$ of predicted risk) or, less frequently, with normal coronary anatomy at ICA. ${ }^{15,16}$ Both strategies for the definitions of normal limits databases of 
myocardial perfusion have relevant drawbacks and limitations. On one hand, the selection of patients at exceedingly low risk of CAD may identify only an extreme of the distribution of normal patients, theoretically limiting the applicability of those data to a more vast population of subjects. On the other hand, the selection of subjects with normal coronary arteries at ICA may pose the risk to include also patients with different cardiac pathologies, such as cardiomyopathies ${ }^{17}$ or coronary microvascular dysfunction due to cardiovascular risk factors. ${ }^{6}$ Moreover, the majority of the established normal limits databases have been created using typically standard SPECT cameras and may not be directly applied to novel dedicated cardiac devices.

As a matter of fact, in the last years, nuclear cardiology has been characterized by relevant technological advances, such as the introduction of dedicated cardiac cameras equipped with solid-state cadmium-zinc-telluride (CZT) detectors, characterized by significantly increased photon sensitivity and spatial resolution compared to traditional SPECT devices. ${ }^{18}$ Because of their dedicated design, CZT cameras allow to radically reduce the injected radionuclide dose while maintaining an excellent image quality. Accordingly, initial reports have clearly confirmed the excellent diagnostic accuracy of CZT cameras in detecting CAD with a global radiation burden as low as 1-2 mSv per study. ${ }^{19,20}$

In most of these reports, evaluation of myocardial perfusion was performed visually or based on a local normal limits database, since the majority of the vendorsupplied normal limits databases for the automatic semiquantification of myocardial ischemic burden are not validated for CZT devices.

The clinical relevance of this apparent limitation of the currently used SPECT software was addressed by Gregoire et al and is reported in the present number of the Journal of Nuclear Cardiology. ${ }^{21}$

This study investigated the need of implementing the existing vendor-supplied normal limits databases with local center data to increase the accuracy in detecting CAD in patients submitted to MPI on a dedicated high-end CZT cardiac camera with ${ }^{201} \mathrm{Tl}$ as a perfusion tracer. In order to accomplish this task, the accuracy of an already well-validated quantification perfusion SPECT software (QPS, Cedar-Sinai, Los Angeles, CA) was tested first using the vendor-supplied normal limits database for the automatic quantification of myocardial ischemia, and then after implementing local population-specific normal limits. Specifically, a population of 65 patients (30 males) at relatively low risk of CAD after non-invasive evaluation and with visually normal myocardial perfusion at stress/rest single-day CZT was used to create a local normal limits database for myocardial perfusion. Thereafter, the accuracy of this normal limits database in detecting significant CAD was tested in a second, slightly larger, population of 170 patients subjected to ${ }^{201} \mathrm{Tl} \mathrm{CZT}$ and ICA \pm FFR and without other major confounding factor. In each patient, the total perfusion deficit (TPD) was computed from stress and rest CZT images and the ischemic TPD (i-TPD) was derived as a measure of the global myocardial ischemic burden using both the vendor-supplied and the local population-specific normal limits databases. Moreover, in order to perform a per-vessel analysis, the vessel-based summed difference score (SDS) was also computed for each coronary territory. The parameters obtained with the automatic quantification of myocardial ischemic burden at CZT (i.e., i-TPD and SDS) showed a similar accuracy in unmasking the presence of significant CAD, independently from the employed normal limits database (vendor-supplied vs local). As a matter of fact, despite the existence of only a moderate correlation between the values of i-TPD obtained with the two databases $(r=0.58)$, the implementation of the locally derived, camera and population-specific, normal limits database for myocardial perfusion did not add any relevant diagnostic benefit over the vendorsupplied ones. These data were further confirmed both in the per-patient analysis, with accuracies of CZT imaging of roughly $70 \%$, and in the vessel-based analysis, with accuracies in detecting CAD in the range of $65 \%$ to $70 \%$.

The relatively surprising results of this study point the accent on a number of questions that should be carefully addressed. First of all, the extremely limited penetration of FFR assessment in the case of coronary lesions of intermediate angiographic severity makes, once again, the gold standard used for the evaluation of CZT accuracy mainly anatomical, thus creating a relevant diagnostic bias. Moreover, in the study by Gregoire et al, the majority of the supposedly healthy subjects used to create the local normal limits database of myocardial perfusion data were symptomatic for chest pain and presented a still significant pre-test probability of CAD $(<35 \%)$, theoretically limiting the applicability of those data especially when a mainly anatomical gold standard, such as ICA, is used. Finally, the accuracies of CZT imaging reported in the present study are lower than expected, especially in the vessel-based analysis. In fact, other experiences, using mainly visual analysis of perfusion images, have reported higher accuracies of CZT imaging in detecting CAD with sensitivities and specificities in the range of $70 \%$ to $80 \% .^{13,22}$ Therefore, a direct comparison of the accuracies of automatic vs visual analysis of CZT perfusion images in a larger patients' population is needed. 
Despite these limitations, those results clearly highlight the extreme robustness and reproducibility of the diagnostic information that can be automatically obtained with modern perfusion imaging. In fact, almost independently of the stress test used (i.e., physical, pharmacological, or both), the radiotracer injected (i.e., ${ }^{99 \mathrm{~m}} \mathrm{Tc}$ radiolabelled tracers or ${ }^{201} \mathrm{Tl}$ ), the camera employed (i.e., traditional SPECT camera vs CZT dedicated cardiac device), and the normal limits database used (i.e., vendor-supplied vs local), the software-based automatic quantification of myocardial ischemia offers the chance to obtain a rapid assessment of global ischemic burden and to unmask the presence of significant $\mathrm{CAD}$ with an acceptable accuracy. Interestingly, the reported accuracy of CZT cameras is similar to that obtained with traditional SPECT devices, which is in contrast with previous experiences and should be further addressed in larger comparative studies.

In fact, only a well-powered prospective study using a robust and widely accepted reference for CAD hemodynamic significance, such as ICA + FFR, can answer this question, giving the chance to definitively downgrade the relevance of the previous evidence based on only anatomical references. Finally, the study by Gregoire et al underscores how some of the peculiar strengths of CZT imaging are still infrequently exploited, possibly limiting the diffusion of CZT imaging in clinical practice. In particular, these high-end cameras allow a radical reduction of the radiotracer's injected dose, with a significant decrease of the test's global radiation burden, ${ }^{13,20,22}$ making CZT imaging the new reference standard for MPI in current clinical practice.

In conclusion, the study by Gregoire et al, brings other solid evidence in favor of the diffusion of CZT imaging in everyday clinical practice, further underscoring its overall robustness, accuracy, and versatility, which have made this imaging modality as the new state-of-the-art technique for the non-invasive evaluation of myocardial perfusion.

Disclosure Alessia Gimelli and Riccardo Liga have no conflicts of interest to disclose.

\section{References}

1. Uren NG, Melin JA, De Bruyne B, Wijns W, Baudhuin T, Camici PG. Relation between myocardial blood flow and the severity of coronary-artery stenosis. N Engl J Med 1994;330:1782-8.

2. Gould KL, Johnson NP, Bateman TM, Beanlands RS, Bengel FM, Bober R, et al. Anatomic versus physiologic assessment of coronary artery disease. Role of coronary flow reserve, fractional flow reserve, and positron emission tomography imaging in revascularization decision-making. J Am Coll Cardiol 2013;62:1639-53.

3. Kapur A, Latus KA, Davies G, Dhawan RT, Eastick S, Jarritt PH, et al. A comparison of three radionuclide myocardial perfusion tracers in clinical practice: The ROBUST study. Eur J Nucl Med Mol Imaging 2002;29:1608-16.

4. Picano E, Molinaro S, Pasanisi E. The diagnostic accuracy of pharmacological stress echocardiography for the assessment of coronary artery disease: A meta-analysis. Cardiovasc Ultrasound 2008;6:30.

5. Greenwood JP, Maredia N, Younger JF, Brown JM, Nixon J, Everett CC, et al. Cardiovascular magnetic resonance and singlephoton emission computed tomography for diagnosis of coronary heart disease (CE-MARC): A prospective trial. Lancet 2012;379:453-60

6. Crea F, Camici PG, Bairey Merz CN. Coronary microvascular dysfunction: An update. Eur Heart J 2014;35:1101-11.

7. Liga R, Marini C, Coceani M, Filidei E, Schlueter M, Bianchi M, et al. Structural abnormalities of the coronary arterial wall-in addition to luminal narrowing-affect myocardial blood flow reserve. J Nucl Med 2011;52:1704-12.

8. Naya M, Murthy VL, Blankstein R, Sitek A, Hainer J, Foster C, et al. Quantitative relationship between the extent and morphology of coronary atherosclerotic plaque and downstream myocardial perfusion. J Am Coll Cardiol 2011;58:1807-16.

9. De Bruyne B, Fearon WF, Pijls NH, Barbato E, Tonino P, Piroth $\mathrm{Z}$, et al. Fractional flow reserve-guided PCI for stable coronary artery disease. N Engl J Med 2014;371:1208-17.

10. Rochitte CE, George RT, Chen MY, Arbab-Zadeh A, Dewey M, Miller JM, et al. Computed tomography angiography and perfusion to assess coronary artery stenosis causing perfusion defects by single photon emission computed tomography: The CORE320 study. Eur Heart J 2014;35:1120-30.

11. Neglia D, Rovai D, Caselli C, Pietila M, Teresinska A, AguadéBruix S, et al. Detection of significant coronary artery disease by noninvasive anatomical and functional imaging. Circ Cardiovasc Imaging 2015;8:e02179. doi: 10.1161/CIRCIMAGING.114.002179.

12. Kajander S, Joutsiniemi E, Saraste M, Pietilä M, Ukkonen H, Saraste A, et al. Cardiac positron emission tomography/computed tomography imaging accurately detects anatomically and functionally significant coronary artery disease. Circulation 2010;122:603-13.

13. Gimelli A, Liga R, Pasanisi EM, Casagranda M, Coceani M, Marzullo P. Influence of cardiac stress protocol on myocardial perfusion imaging accuracy: The role of exercise level on the evaluation of ischemic burden. J Nucl Cardiol 2015

14. Arsanjani R, Xu Y, Hayes SW, Fish M, Lemley M Jr, Gerlach J, et al. Comparison of fully automated computer analysis and visual scoring for detection of coronary artery disease from myocardial perfusion SPECT in a large population. J Nucl Med 2013;54:221-8.

15. Toft J, Lindahl D, Ohlsson M, Palmer J, Lundin A, Edenbrandt L, et al. The optimal reference population for cardiac normality in myocardial SPET in the detection of coronary artery stenoses: Patients with normal coronary angiography or subjects with low likelihood of coronary artery disease? Eur $\mathrm{J}$ Nucl Med 2001;28:831-5.

16. Guner LA, Karabacak NI, Cakir T, Akdemir OU, Kocaman SA, Cengel A, et al. Comparison of diagnostic performances of three different software packages in detecting coronary artery disease. Eur J Nucl Med Mol Imaging 2010;37:2070-8.

17. Neglia D, Parodi O, Gallopin M, Sambuceti G, Giorgetti A, Pratali $\mathrm{L}$, et al. Myocardial blood flow response to pacing tachycardia and to dipyridamole infusion in patients with dilated cardiomyopathy without overt heart failure. A quantitative assessment by positron emission tomography. Circulation 1995;92:796-804.

18. Imbert L, Poussier S, Franken PR, Songy B, Verger A, Morel O, et al. Compared performance of high-sensitivity cameras dedicated 
to myocardial perfusion SPECT: A comprehensive analysis of phantom and human images. J Nucl Med 2012;53:1897-903.

19. Gimelli A, Bottai M, Giorgetti A, Genovesi D, Kusch A, Ripoli A, et al. Comparison between ultrafast and standard single-photon emission CT in patients with coronary artery disease: A pilot study. Circ Cardiovasc Imaging 2011;4:51-8.

20. Einstein AJ, Blankstein R, Andrews H, Fish M, Padgett R, Hayes SW, et al. Comparison of image quality, myocardial perfusion, and left ventricular function between standard imaging and single-injection ultra-low-dose imaging using a high-efficiency SPECT camera: The MILLISIEVERT study. J Nucl Med 2014;55: 1430-7.

21. Gregoire B, Pina-Jomir G, Bontemps L, Janier M, Scheiber C. The value of local normal limits in quantitative Thallium-201 CZT MPI SPECT. J Nucl Cardiol 2016

22. Sharir T, Pinskiy M, Pardes A, Rochman A, Prokhorov V, Kovalski G, et al. Comparison of the diagnostic accuracies of very low stress-dose with standard-dose myocardial perfusion imaging: Automated quantification of one-day, stress-first SPECT using a CZT camera. J Nucl Cardiol 2016;23:11-20. 